\title{
CONFINAMIENTO POR COVID-19: ¿CÓMO HA IMPACTADO EN EL CONSUMO DE VINO Y EN LAS ACTIVIDADES DE ENOTURISMO?
}

\author{
África Martínez-Povedaa*, Asunción María Agulló Torres ${ }^{\mathrm{a}}$, Margarita Brugarolas ${ }^{\mathrm{a}}$, Laura \\ Martínez-Carrasco $^{\mathrm{a}}$ \\ ${ }^{a}$ Universidad Miguel Hernández,(Orihuela, africa.martinez@umh.es)
}

\section{Resumen}

La declaración de pandemia mundial por la COVID-19 ha tenido efectos muy negativos en multitud de sectores económicos. El sector de la hostelería y el turismo no ha sido ajeno a esta situación. El consumo moderado de vino se ha considerado saludable, no tanto por los beneficios que reporta su ingesta, sino como factor de socialización. Las actividades de enoturismo tienen esta componente social, ya que conjugan el consumo de vino con una actividad lúdica, teniendo como consecuencia positiva la visita a bodegas, el conocimiento de una DO, el territorio, el paisaje... Sin embargo, la pandemia ha supuesto un cambio de guion en el hábito de consumo de vino y disfrute de actividades enoturísticas ya que estas acciones no se han podido realizar. En el presente trabajo se realizó una encuesta on line a 365 individuos para determinar el cambio de hábitos durante el confinamiento tanto de consumo como de las nuevas actividades enoturísticas propuestas, y si esto ha provocado una intención de consumo distinta para el futuro. El $76 \%$ ha realizado algunas de las posibles propuestas on line de enoturismo y a más del $80 \%$ les gustaría realizar enoturismo finalizado el confinamiento lo que muestra el creciente interés por dichas actividades.

Palabras clave: consumo de vino, COVID-19, enoturismo.

\section{Introducción y objetivos}

La situación provocada por el confinamiento debido a la COVID-19, ha generado un efecto negativo en multitud de sectores. El sector vitivinícola no ha quedado al margen de esta situación, y tras la recuperación y posterior estabilización del consumo de vino en la última década, esta situación ha sufrido un cambio en los últimos meses, modificando los hábitos de compra y consumo de vino.

Está reconocido que el consumo moderado junto con las actividades sociales llevadas a cabo gracias al efecto socializador del vino hacen que mejore la salud de los consumidores (Li, 2020). Según Scott et al. (2018), el consumo moderado de alcohol invita a la oportunidad de interacción social, por tanto las ventajas para la salud no son únicamente debidas al consumo moderado de vino, sino al modelo en que se consume. Los pilares fundamentales del estilo de vida mediterráneo incluyen además de estar activo y alimentarse correctamente, disfrutar de la socialización, es decir, un consumo moderado, que habitualmente se realiza en familia o en compañia (FIVIN, 2020).

El consumo de vino en España realizado en el canal de la hostelería y la restauración supone, en volumen, más de un tercio de las ventas de vino en España (OIVE, 2020). Pero, junto a estos canales tradicionales, existen otros como las ventas a través de clubs de vino, las ventas en vinotecas y tiendas gourmets, las ventas on line no incluidas en los establecimientos de alimentación y las ventas directas de bodega y/o generadas por las actividades enoturísticas (OIVE, 2020), que también se han visto grandemente perjudicadas por la pandemia. Uno de los canales que a priori se ha visto beneficiado es el canal de venta online (ALIMARKET, 2020).

El otro canal que está tomando un auge cada vez mayor es el enoturismo. Pero este, no son sólo relaciones públicas y más ventas para la bodega, recoge otros factores amparados bajo el paraguas del turismo y la hospitalidad (Woldarsky, 2020). Sin embargo, la pandemia ha creado la necesidad de buscar nuevos momentos para el consumo de vino gracias a las tecnologías de comunicación. De hecho, las bodegas no han renunciado al componente social que tiene la unión de vino y turismo y se han reinventado creando experiencias online a la espera de tiempos mejores. La organización de catas y visitas virtuales, seminarios de formación online, charlas entorno a una copa de vino, etc..., han mantenido vivas las experiencias enoturísticas durante el confinamiento. Además, el turismo enológico ha encontrado la capacidad de promover el consumo de vino, haciendo del confinamiento en los hogares una estrategia para promover el consumo del vino a la espera de poder volver a visitar las bodegas y compartir actividades presenciales en torno a una copa de vino (Arellano, 2020).

El objetivo del presente estudio es analizar el consumo de vino durante el confinamiento y detectar las nuevas actividades de socialización entorno a una copa de vino.

\section{Metodología}


En este estudio se realizaron 365 encuestas entre los meses de mayo y agosto de 2020 a través de Google Formularios. La difusion de la encuesta comenzó en redes sociales a partir de grupos relacionados con el vino y la gastronomía. El cuestionario estaba dividido en tres bloques centrados en el consumo de vino, actividades entorno al vino y el perfil del consumidor. En el primer bloque, el encuestado debía responder a sus habitos de compra y consumo, antes y durante el confinamiento, debido a la COVID-19. En el segundo bloque, los encuestados debían responder sobre las actividades de enoturismo realizadas previas al confimiento y los nuevos modelos de actividades aparecidas durante el confinamiento. Por último, para caracterizar a la muestra se planteó un tercer bloque con preguntas relacionadas con el tipo de vino consumido, conocimento sobre el vino y variables sociodemográficas.

\section{Resultados}

De los 365 individuos que accedieron a realizar la encuesta, se descartaron el 19,7\% por no ser compradores y/o consumidores de vino. Por tanto la muestra final que realizó la encuesta completa fue de 293 individuos. En primer lugar se preguntó a los encuestados si habitualmente compraban o consumían diferentes bebidas alcohólicas. El 96,6\% afirmaron que consumían vino, bajando este porcentaje en el caso de la cerveza $(87,7 \%)$, resultando el resto de bebidas alchólicas preguntadas con un porcentaje mucho menor. En cuanto a la frecuencia de consumo, el 75,1\% de los consultados consumen vino al menos una vez a la semana en el hogar, frente al 70,3\% que lo consumen fuera del hogar con esa misma frecuencia.

Para analizar si la COVID-19 había generado cambios en los hábitos de compra y consumo se hicieron diferentes preguntas. Los resultado obtenidos muestran que, durante el confinamiento, el 51,5\% de los encuestados, afirma que ha consumido más vino que en condiciones normales, además, el 36,6\% ha consumido vino con una frecuencia mayor a la habitual.

En su conjunto, el gasto del vino consumido en el hogar antes y durante el confinamiento es similar como se puede apreciar en el gráfico 1. Pero, si analizamos los tipos de vinos en función de su precio, se observa que el consumo de vinos de precio menor a $5 €$ ha disminuido un $32 \%$ y sin embargo se ha incrementado el consumo de vinos de precio de más de $30 €$ en un $66 \%$.

Gráfico 1. ¿Qué promedio gastaba a la semana en comprar VINO para consumir en el hogar antes y durante el confinamiento?

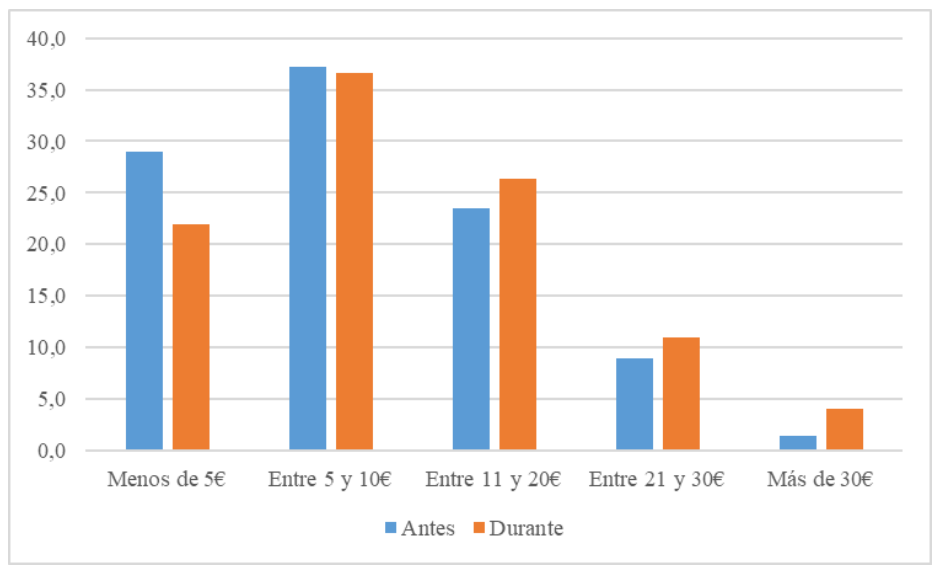

Fuente: Elaboración propia

En cuanto a los lugares de compra habitual, antes del confinamiento las ventas on line sólo representaban el $4,8 \%$ en su conjunto. Con la pandemia el porcentaje de venta online ha sido lógicamente superior, destacando el incremento hasta cerca del $20 \%$ en la distribucion a través de tiendas especializadas y bodegas (gráfico 2).

Para comprobar el grado de socialización que tiene el consumo de vino, se pidió a los encuestados que valorasen el consumo de vino en diferentes momentos en una escala de 5 niveles, siendo 1 nunca lo consumía y 5 siempre lo consumía. Como se puede observar en el gráfico 3, la respuesta más señalada fue "cuando salgo a comer/cenar fuera de casa", con una puntuación media de 3,90, seguida de "en reuniones de amigos y familiares" $(3,71)$. El resto de ocasiones, todas ellas relacionadas con un consumo en el hogar obtuvieron una puntuación media cercana a 3 o inferior. 
Gráfico 2. Distribuión de las ventas de vino (en \%) en diferentes canales

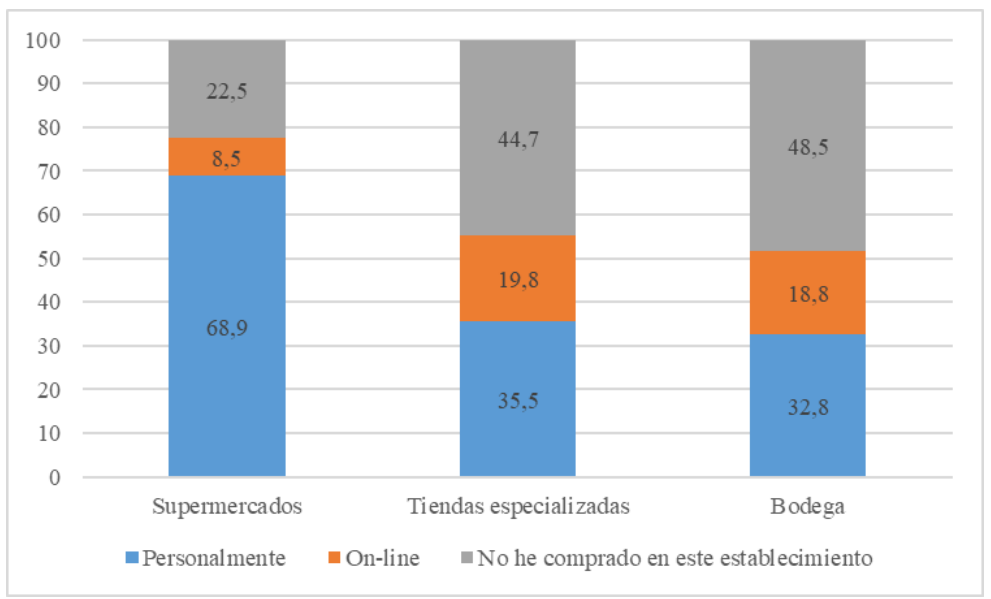

Fuente: Elaboración propia

Gráfico 3. Antes del confinamiento, ¿Con qué frecuencia consumía VINO en las distintas ocasiones mostradas? (1. Nunca - 5. Siempre)

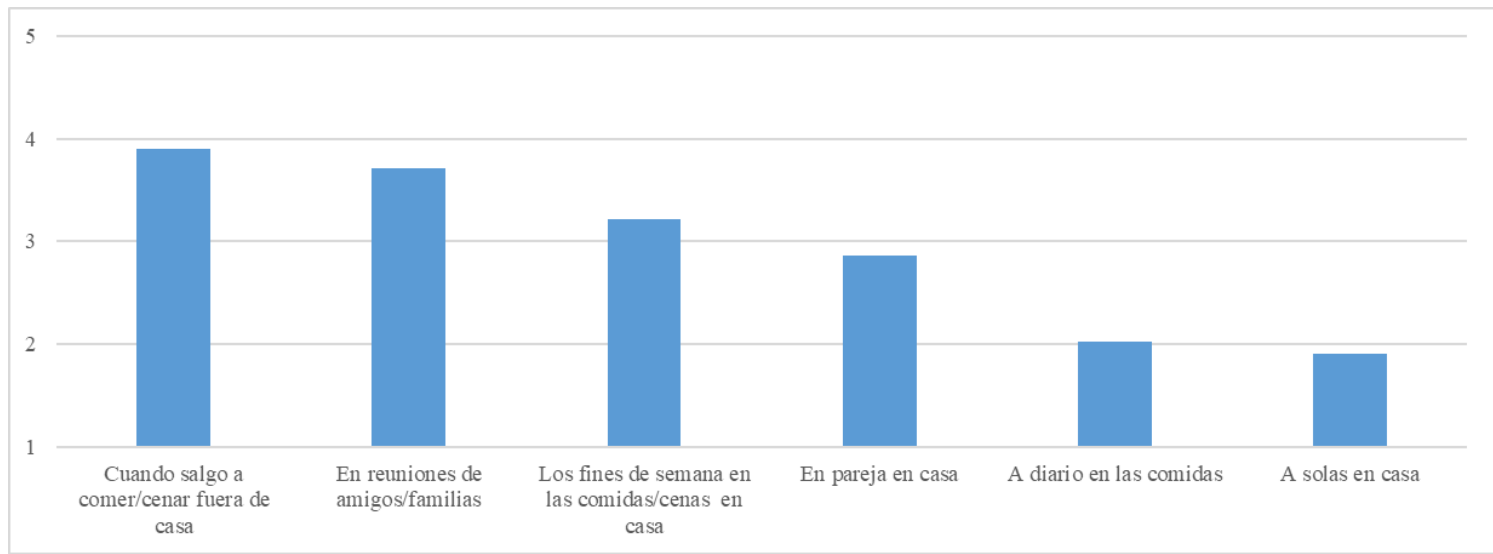

Fuente: Elaboración propia

En cuanto a las actividades de enoturismo antes de la COVID-19, la "visita a bodega y cata de vino" fué las más señalada $(71,2 \%$ de los casos) seguida de "cata de vino con maridaje" y "visita a viñedo" señalada por el $53,1 \%$ y $45,2 \%$ de los casos respectivamente. Entre las opciones más señaladas durante el confinamiento fueron "cocinar con vino" $(55,7 \%)$, "realizar vinollamadas para quedar con familiares y/o amigos y disfrutar del vino" (39\%) y "leer con una copa de vino" $(26,1 \%)$. También han surgido nuevas actividades creadas por las bodegas para suplir la presencialidad en las mismas. En el cuadro 1 se muestran los porcentajes de las activiades señaladas en función de distintas opciones mostradas a los consumidores.

Cuadro 1. Actividades realizadas antes y durante el confinamiento

\begin{tabular}{|l|l|}
\hline \multicolumn{1}{|c|}{ Actividades de enoturismo pre-COVID-19 } \\
\hline P1.Visita a bodega y cata de vino & $\mathbf{7 1 , 2 \%}$ \\
P2. Cata de vino con maridaje & $53,1 \%$ \\
P3. Visita a viñedos & $45,2 \%$ \\
P4. Visita a museo enológico & $30,8 \%$ \\
P5. Seguir actividades sobre el vino en redes sociales & $36,0 \%$ \\
\hline \multicolumn{2}{|c|}{ Nuevas actividades sociales durante la-COVID-19 } \\
\hline D1. Catas virtuales en directo Actividades individuales relacionadas con el consumo de vino durante la COVID-19 \\
D2. Realizar "Vinollamadas" para quedar con familiares y/o amigos y disfrutar del vino \\
D3. Realizar visitas virtuales a bodegas & $\mathbf{3 9 , 1 \%}$ \\
D4. Asistir a charlas sobre vinos y/o bodegas en redes sociales & $3,8 \%$ \\
D5. Realizar cursos relacionados con el mundo y la cultura del vino on line & $\mathbf{1 6 , 0 \%}$ \\
\hline \multicolumn{2}{|c|}{$\mathbf{5 5 , 7 \%}$} \\
\hline D6. Cocinar con vino & $26,1 \%$ \\
D7. Leer con una copa de vino & $25,4 \%$ \\
D8. Ver películas, documentales y/o series relacionadas con el mundo del vino & \\
\hline
\end{tabular}


La muestra se ha segmentado en función del conocimiento sobre vino que mostraban los encuestados. En el gráfico 4, se muestran los porcentajes de individuos de cada segmento que han realizado las actividades propuestas durante el confinamiento.

Gráfico 4. Actividades realizadas durante el confinamiento (en \%)

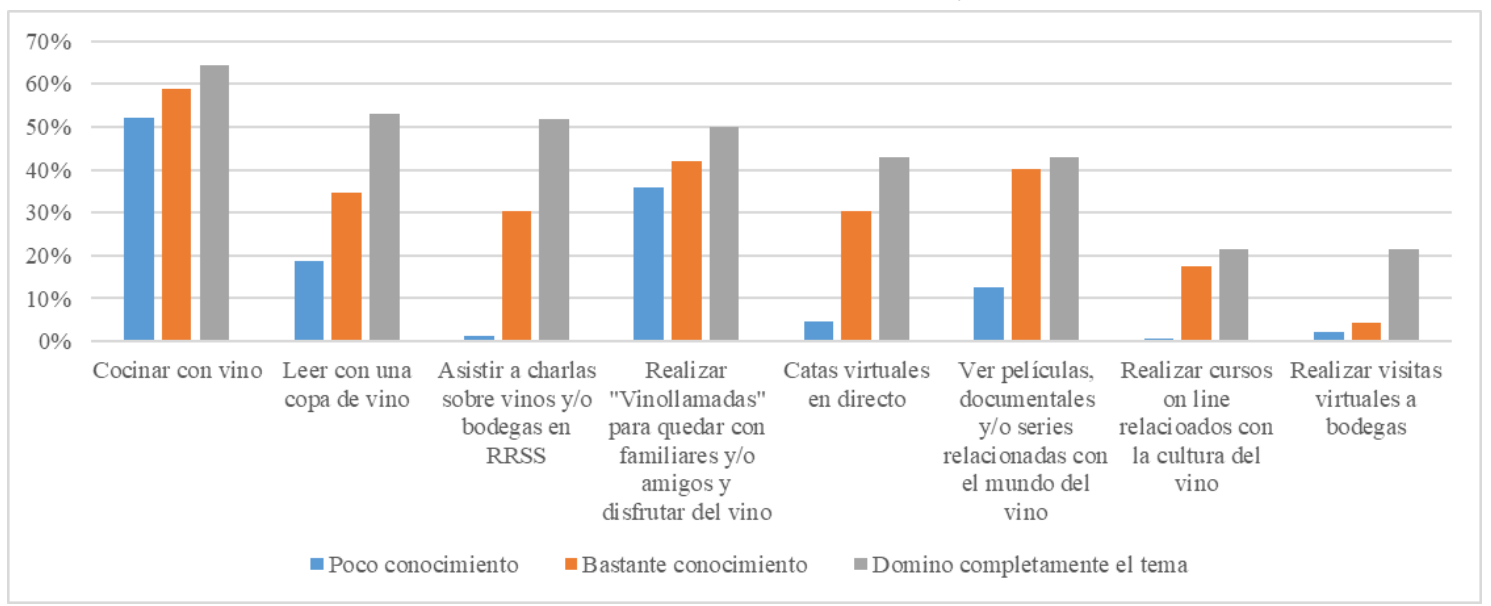

Fuente: Elaboración propia

\section{Conclusiones}

La pandemia por la COVID-19 ha afectado duramente al sector de la hostelería y en este sentido las bodegas se han visto muy perjudicadas ya que un tercio del consumo se realiza fuera del hogar. El consumo en el hogar no solo ha aumentado, sino que también lo ha hecho el gasto efectuado durante la COVID-19, al consumirse vinos con mayor precio unitario. Esto ha ayudado a paliar las pérdidas del sector.

El gran esfuerzo de las bodegas por mantener contacto con los enoturistas (D1, D3, D4 y D5), no se ha visto recompensado, puesto que las opciones creadas para suplir las actividades de socialización in situ en las bodegas no han tenido mucha repercusión durante el confinamiento en términos globales, aunque si ha sido muy aceptada entre el grupo de encuestados con dominio en la materia. Las actividades online, fueron de las menos señaladas antes de la COVID-19, y a pesar del confinamiento la aceptación en este tipo de actividades no mejoró. El acercamiento a la cultura del vino pasa por una formación presencial donde el cosumidor pueda disfrutar de la cultura y cata del vino en compañia, corroborando el caracter social que tiene el vino.

\section{Bibliografía}

ALIMARKET (2020). Las ventas de vino podrían caer hasta un $40 \%$ este año por el cierre de la hostelería. https://www.alimarket.es/alimentacion/revista

Arellano, J. R., Santoyo, M., Jiménez, B. (2020). "Los procesos de socialización del consumo de vino de mesa en México y los estilos culturales de vida". En Sarmiento, F.F., Valles, M.C. y Mota, V.E. (Eds.): Factores críticos y estratégicos en la interacción territorial desafíos actuales y escenarios futuros. Universidad Nacional Autónoma de México y Asociación Mexicana de Ciencias para el Desarrollo Regional: 527-538.

FIVIN (2020). Fundación para la investigación del vino y la nutrición. http://www.fivin.com/

Li, Y., Schoufour, J., Wang, D.D., Dhana, K., Pan, A., Liu, X., Song, M., Liu, G., Shin, H.J., Sun, Q., AlShaar, L., Wang, M., Rimm, E.B., Hertzmark, E., Stampfer, M.J., Willett, W.C., Franco, O.H. y Hu1, F.B. (2020). "Healthy lifestyle and life expectancy free of cancer, cardiovascular disease, and type 2 diabetes: prospective cohort study". BMJ, 368: 16669.

OIVE (2020). Radiografía de canales de consumo de vinos en España. Interprofesional del Vino de España. Madrid, España.

Scott, R.G., Wiener, C.H., Paulson, D. (2020). "The Benefit of Moderate Alcohol Use on Mood and Functional Ability in Later Life: Due to Beers or Frequent Cheers?” The Gerontologist, 60(1):8088.

Woldarsky, V. (2020) "Buenas prácticas en enoturismo. Mejorar la calidad del servicio y optimizar la experiencia del visitante”. En Compés, R y Szolnoki, G (Eds.): Enoturismo sostenible e innovador. Modelos de éxito alrededor del mundo. Cajamar Caja Rural: 35-50. 